\title{
EXTERNAL PULSED PLASMA PROPULSION (EPPP) ANALYSIS MATURATION ${ }^{+}$
}

\author{
Joseph A. Bonometti and P. Jeff Morton" \\ NASA Marshall Space Flight Center \\ Propulsion Research Center. TD-40 \\ Huntsville. Alabama 35812 \\ (256) $544-4019$ and (256) 544-4613
}

\section{ABSTRACT}

External Pulsed Plasma Propulsion (EPPP) systems are at the stage of engineering infancy with evolving paradigms for application. performance and general characteristics. Recent efforts have focused on an approach that employs existing technologies with near term EPPP development for usage in interplanetary exploration and asteroid/comet deflection. if mandated. The inherent advantages of EPPP are discussed and its application to a variety of propulsion concepts is explored. These include, but are not limited to. utilizing energy sources such as fission. fusion and antimatter, as well as. improved chemical explosives. A mars mission scenario is presented as a demonstration of its capability using existing technologies. A suggested alternate means to improve EPPP efficiencies could also lead to a heavy lift (non-nuclear) launch vehicle capability. Conceivably, true low-cost, access to space is possible using advanced explosive propellants and/or coupling the EPPP vehicle to a "beam propellant" concept. EPPP systems appear to offer an approach that can potentially cover ETO through interstellar transportation capability. A technology roadmap is presented that shows mutual benefits pertaining to a substantial number of existing space propulsion and research areas.

\section{INTRODUCTION}

External Pulsed Plasma Propulsion (EPPP) is a highly efficient method of propulsion. No matter what energy source is used to generate the plasma burst, there are inherent advantages to its operation. EPPPs primary advantage is that it reduces the material temperature constraint innate in all

\footnotetext{
* Propulsion Research Engineer. MSFC Propulsion Research (enter

+ This paper is declared a work of the U.S. Government and is not subject to

copyright protection in the United States.
}

conventional thermal rocket engines. In most liquid chemical engines, using a combustion chamber and an exhaust nozzle. a large amount of fuel is used to convectively cool the chamber walls. Without which. the walls would quickly reach their melting temperature. The same is true for nuclear thermal concepts (solid core) where the fuel temperature must be maintained below its melting point. In practice. the temperature is further curbed by lower material yeld strengths at higher temperatures and practical engineering safety margins. Even in a gas core nuclear reactor. the fuel temperature cannot be left unchecked as the containment walls. operating in steady state must be actively cooled to avoid exceeding practical material limitations.

The material limit paradox is that an arbitrary hot central core must be separated by a physical barrier. If the barrier is a solid. it will conduct the energy when the core is in contact with it. If the core is not in contact (e.g. by some high-speed flow effect or electromagnetic effects) then the core's radiative energy is still absorbed or transmitted by the wall. In the transmitted case, there must be a second nontransparent wall or the hot core will be radiating into space (a 4 Kelvin heat sink). In these radiative cases, the core temperature to the fourth power dominates the heat loss from the system. 'Assuming material temperatures are limited to roughly $3.000 \mathrm{Kelvin}$. then even a 5,000 Kelvin gas temperature generates a huge 30 $\mathrm{MW} / \mathrm{m}^{\wedge} 2$ of waste heat (through radiation alone) that must be rejected. Depending on the particular configuration and gas properties, that is the same order-of-magnitude as the energy needed to heat the gas (i.e. approximately $5 \mathrm{MW} / \mathrm{kg}$ to ideally heat a gas from 300 Kelvin to 5.000 Kelvin. which even for a pressure of 1.000 atmospheres is about $15 \mathrm{MW} / \mathrm{kg}$ to reject). Obviously, the actual temperature, pressure and heat fluxes would be different at equilibrium in a real engine. Nevertheless. the equilibrium performance is only further degraded.

Another discouraging fact is that even an ideal radiator requires an order-of-magnitude greater surface area to radiantly reject the heat into space. Both these problems become worst as the gas temperature rises. In all realistic cases. known 
material temperature limits are exceeded in any steady state operation without active heat rejection. This almost always requires using large amounts of cold gas as film cooling. That immediately reduces the engine"s Specific Impulse (Isp). while adding complexity and cost to the system.

The solution is to operate the hottest part of the engine external to the confinement walls. In the pulsed fission rocket. the "containment chamber" or engine walls are allowed to "melt" (more precisely vaporize into a plasma) with each pulse and no limitation is imposed. This would suffer the same thermal limitation on the rest of the propulsion unit if the engine were not then pulsed. Thus, the same quantity of energy is liberated. but in a exceedingly short duration. Since thermal heating processes are relatively slow. propagating at the speed of sound in a material. the heating is minimal, if the energy is released at a much faster rate. This implies the pulse be an "explosion" and propagate at mach numbers much greater than one. as is the case for conventional explosives and supercritical nuclear reactions.

In the case of self-contained pulse units, the entire energy generation mechanism is consumed in the plasma pulse process. It would be comparable to using the structural tankage of conventional chemical propellant systems and exhausting it out the engine as thrust. That is unlike most space propulsion drives (including most rusion. antimatter and various forms of MHD) where the bulk of the vehicle mass is the engine. Fast fission processes are also unique in the fact that the vast majority of the energy released is directed to the exhaust velocity of the propellant. There is no thermal conversion loss (as in a nuclear thermal or electric system that must actively reject waste heat) and only minimum loss of energy. due to it not being in a usable propulsion form (i.e. fusion or antimatter).

\section{BACKGROUND}

EPPP is a descendant of the Air Force's 1958 ORION project, but with substantial differences. In the GABRIEL series, an evolutionary framework of EPPP concepts, the vehicles are only for interplanetary exploration and asteroid/comet defense missions where Earth contamination is not an issue. In the initial concept resurgence one-year ago, the vehicles were conjectured to be small. slow pulsing and use existing materials and technologies. " A somewhat subjective goal of 5.000 seconds Isp was set as the minimum performance that would be necessary to overcome political objections of self-contained nuclear pulse units (although very different from a nuclear weapon as seen from Table 1 , the mere use of fissionable material is objectionable to some, even when it is for permanent disposal). The small size of the pusher plate (limited to 5 meters to keep it within existing launch vehicle shroud sizes) closed the design parameter space outside the predefined limits. Falling far short of the desired 5.000 seconds Isp. the GABRIEL vehicle needed an alternate approach. since it appeared that the pulse unit mass could not be reduced nor directionalized sufficiently to compensate for the restricted plate size.

\section{TABLE I: CHARACTERISTIC COMPARISONS}

\begin{tabular}{|c|c|c|}
\hline POSITIVE CHARACTERISTICS & EPPP & BONB \\
\hline Peaceful Purpose & Yos & No \\
\hline Reduces Risk of Radiation Exposure to Humans & Yes & No \\
\hline The Lower the Y'ield the More Desirable & Yos & No \\
\hline $\begin{array}{l}\text { Fail Safe to Only be Used with Spacecraft Propulsion } \\
\text { System }\end{array}$ & Yes & No \\
\hline Particles Leave Solar System Forever & Yes & No \\
\hline Single, Quick Flash Only & Yes & $\lambda_{0}$ \\
\hline $\begin{array}{l}\text { Permanent Reduction of Nuclear Weapon Vaterial } \\
\text { from Farth }\end{array}$ & Ys & Wo \\
\hline
\end{tabular}

\begin{tabular}{|l|c|c|}
\hline \multicolumn{1}{|c|}{ NEGATIVE CHARACTERISTICS } & EPPP & BON1B \\
\hline Normally over I Kiloton Yield & No & Yes \\
\hline Contamination of Environment & No & Yes \\
\hline Radiation Enhancement Desired & No & Yes \\
\hline Neutron Enhancement Desired & No & Yes \\
\hline Mushroom Cloud or Blast Wave & No & Yes \\
\hline E.MP Effects & No & Yes \\
\hline Harmful to Earth & Vo & Yes \\
\hline
\end{tabular}

\begin{tabular}{|l|c|c|}
\hline \multicolumn{1}{|c|}{ NEl TRAL. CHARACTERISTICS } & EPPP & BOIIB \\
\hline liberates Vuclear Fnergy & Yu & Ye \\
\hline Could he Self-c contained & Yu & Ys \\
\hline High Energy to Mass Ratio & Yes & Yes \\
\hline Phisics Developed From Weapons Research & Yes & Yes \\
\hline Known Technology & Yes & Yes \\
\hline Fusion Enhanced Possible & Yes & Yes \\
\hline
\end{tabular}

Increasing the effective plate size. or the area over which the plasma acts upon the vehicle. was explored in a variety of ways. In brief. three ways were considered. First, was to look at other EPPP configurations. MEDUSA was considered as well as spinning cables and areoshield mechcanisms. "In all these, the structure size was on the order of kilometers and the mass was large as well. Although solar sail and tether technologies could be borrowed, the technology was not considered practical, particularly in the deployment/control of the large structure. Costs also were a concern, as the mass in LEO was substantial and development would be expensive. Second, was the use of electromagnetic fields where direct plasma contact is limited. This has great potential, but the magnet mass is prohibitive within reasonable development expectations. Also. field strength is inversely proportional to the radius squared. which makes it very difficult to create a field much larger than the ship itself. Concepts, such as MagOrion, appear to be large, costly and rely on major state-ofthe-art improvements or breakthroughs. "If it is feasible to scale down the MagOrion concept sufficiently, this concept has tremendous potential. Some feasibility tests and further analysis is required before this technological path can be explored further (highly encouraged research to be undertaken). The third approach was the most direct. Build a bigger physical plate and either launch it in pieces and or assemble/manufacture it in space or on the Moon. Assembling in space is difficult and very costly, as the International Space Station has shown. Whether assembled or manufactured in 
space (the Moon option was not considered possible because the infrastructure requirements are overwhelming), the completed structure would need to be thoroughly examined with zero defects before it could be placed into operation. Confidence in such final certification would be unacceptably low. Only complete manufacture. inspection and testing on the ground was considered safe and practical for a 10 to 20 meter pusher plate, particularly if composites or advanced alloys were involved.

If the entire ship had to be constructed on the Earth, then launching it would require a new super heavy launch vehicle (as large or larger than the Saturn V) or a cluster of strap-on components (i.e.. 6 to $12 \mathrm{SSME}$ engines under the plate for example). Again expense and complexity makes this unattractive, if the entire EPPP system is considered "payload" to get into orbit. However, if the basic EPPP engine mass could be used in place of conventional engines, plumbing and tankage mass, the need to design a new launch vehicle would be eliminated. The expectation is that additional pulse propellant expense (i.e. some form of advanced chemical explosive or "beamed propellant" methodology) would easily be offset by the design and fielding cost of the additional liquid propellant system. It is this approach that has been adopted for a near-term GABRIEL design.

\section{EARTH-TO-ORBIT CHALLENGE}

The way to propel a large diameter EPPP vehicle to Low Earth Orbit (LEO) without nuclear energy is to carry a chemical explosive equal to oxygen and hydrogen combustion onboard or supply the propellant external to the ship. The latter is generally termed as beamed propellant (an alternate to the beamed energy terminology used for external supplied laser or microwave propelled vehicle concepts). More than likely a combination of advanced chemical explosive and beamed propellant technologies would be employed. A possible Mars mission scenario is presented in the next section that is based on explosive technology slightly less efficient than LOX/LH2, but better than conventional solid rocket motors. Its baseline pusher plate diameter is 16 meters and achieves 5,000 seconds Isp, with nuclear pulse units for interplanetary operation.

Launch costs of such a large vehicle into LEO is somewhat relative. Essentially, it is a one-time event and only must be comparable to the total cost involved in a Mars or similar interplanetary mission. The mass savings on a fast Mars trip ( 7 to 12 months) is enormous when compared to that required for the typical 3-year mission scenario using conventional propulsion. Therefore, even if the system is inefficient for ETO, the gross mission launch weight (all launch vehicle stages, propellant and mission payloads) could be half that of a 3-year mission. And since mission cost is proportional to gross mission mass in order-of-magnitude, it would be a considerable savings.

Related to such financial considerations. are the objections that might be raised to deploy GABRIEL in the first place. The additional costs to handle nuclear material are not significantly higher than other propellants for several reasons.
The infrastructure for such work is in place and is considered a Government "sunk cost" since it is and will be required for civilian and military purposes. Processing pulse units should not incur huge development expense and "volume production" should ease the cost per unit. The expensive fissionable material is considered "excess" and currently incurs costs to store it. Furthermore. plans to decommission the U.S. stockpile would incur even greater expense and still not absolutely assure permanent disposal (as would its expenditure as rocket propellant).

Safety and security are neither major issues nor an overwhelming expense to such a mission. Manned shuttle launches are extremely secure. even from terrorists desiring to "make headlines". The military routinely transports. stores and services thousands of nuclear weapons around the world without incident. These are far more powerful and dangerous than what would be used as pulse units and several steps could be taken to ensure pre-detonation is impossible other than in space behind the vehicle (even handling is safer than for LOX/LH2). Finally. in most other realistic Mars missions. substantial nuclear power in some form must be used and these same risks. costs and political objections must be overcome as well.

\section{Advanced Chemical Explosives}

The energy density content of EPPP explosives should be inherently high. since it can be solid (i.e. more dense than liquids, particularly hydrogen), somewhat self supporting (i.e.. non pressurized tank containment) and demands no pressure/temperature restrictions upon detonation (i.e.. solid rocket motors often have ingredients to control burn rate and pressure sensitivities). Detonations also release the energy quickly, diminishing thermal losses due to minimal interaction time with the vehicle (albeit. a more profound consequence within the fission pulse mode).

Conventional explosives. such as $\mathrm{C}-4$. do not have the energy to perform an ETO mission. Table 2 shows rough energy density for some explosives. Theoretically. there maybe chemical explosives capable of $\mathrm{LOX} / \mathrm{LH} 2$ performance, but at present, that is very speculative. However, obtaining a slightly better performance than existing military's high explosive (i.e., HMX) is probable. Despite drifting into the classified arena, a necessary step for any high powered technology (the best lasers, materials, atomic physics, etc. are military secrets). more powerful compounds probably already exist. Their military deployment might be hampered by uneconomical manufacturing costs, limited storage life or other peculiarity that a one time space launch could accept.

\section{TABLE 2: ENERGY DENSITIES}

\begin{tabular}{|c|c|}
\hline EXPLOSIVE CLASS & NOMIIAL ENERGY DENSITY \\
\hline$\Gamma N T$ & $4 \mathrm{~kJ} / \mathrm{g}$ \\
\hline$(-4$ & $5 \mathrm{~kJ} \mathrm{~g}$ \\
\hline HMX & $6 \mathrm{kJg}$ \\
\hline Speculative Compounds & $75 \mathrm{~kJ} / \mathrm{g}$ \\
\hline Hydrogen/Oxyen & $15 \mathrm{kJg}$ \\
\hline Theoretical Compounds & $+16 \mathrm{KJ} / \mathrm{g}$ \\
\hline
\end{tabular}


The minimum goal for the advanced chemical explosive pulse unit is only to obtain LEO. even if just marginally, with the pusher plate and Momentum Transfer Mechanism (MTM). That is equivalent to lowering the Space Shuttle Isp. but only having to get the engines to LEO (i.e.. no payload. crew, provisions for several weeks on orbit. etc.).

\section{Beamed Propellant}

Being somewhat speculative with the improved propellant performance for the chemical pulse units, the vehicle might still not have enough margin to be practical. Estimated MTM masses might grow. losses in the expanding plasma gas. aerodynamic drag. or other engineering issue may limit performance below the preliminary analysis predicted. An extra margin can be secured by supplemental approaches. The most common is to use solid rocket motors boosters for the initial lift-off. The Space Shuttle uses this concept effectively (the solids provide the large thrust-to-weight ratio lacking in the SSME) and it is employed in the EPPP Mars mission discussed later in this paper. A third stage (LEO insertion) using LOX/LH2 is very difficult due to the added weight. complexity and susceptibility to damage during pulse mode. Its use as a second stage is conceptually easier. as a cluster of Titan or Delta rocket main stages could be bolted together (i.e.. analogous to the Saturn B I) under the plate. Then the entire assembly could be jettisoned at burn out. leaving a minimal insertion to be accomplished with the pulsed third stage. Again. added weight. cost and complexity are major detractors.

The most compatible supplemental approach is to supply the propellant from outside the vehicle. This beamed propellant approach is ideal for EPPP, since no modifications need to be made to the vehicle. The propellant supplied could be from separate rockets. conventional gun batteries. rail guns. mag-lev tracks, or other hypervelocity device. The supply system could be from ground-based infrastructure. aircraft or a combination of both. Space based propellant is also possible, but cost to launch it or manufacture it in space is of questionable economic value (a plausible scenario is as a "virtual" third stage where a small propellant supply, based in orbit, is sent to an ascending EPPP vehicle at its apex to obtain final orbital velocity).

Again, if the object is simply to get the base vehicle to orbit, there appears ample performance margins in a number of futuristic beamed propellant technologies. An example is the Sling-a-tron concept. which appears very adaptable to rapid propellant delivery requirements. Since gravity losses are appreciable during most of an ETO flight, sustained high thrust is required, particularly upon lift-off. Although beamed propellant might be used from the start, a solid rocket initial boost may be very advantageous. It allows an easily controlled start and places the vehicle at a point where ground based batteries or aircraft can readily track the propellant supply point under the vehicle. How far the solid boosters should go into the flight. when the ground based propellant is replaced with airborne propellant beams and how much onboard or spacebased pulse units are utilized will be determined in future optimization studies. Based on expected propellant velocities and reasonable throw weights. it would appear that beamed propellant could only be used in part of the ETO flight envelope. The ground-based beamed propellant must be propelled faster than the vehicle and contend with the dense atmosphere during its flight. At present. practical exit speeds are limited to 3 to $4 \mathrm{~km} / \mathrm{s}$ for most concepts. Therefore, the delta- $\mathrm{V}$ imparted to the ship will only be 2 to $3 \mathrm{~km} / \mathrm{s}$, far short of the 8 or 9 needed to achieve LEO. High flying aircraft may only be able to raise that another $\mathrm{km} / \mathrm{s}$, due to limits on the amount of fuel that can be carried and the altitude achievable by the supply aircraft. Again, future optimization studies will determine these values more precisely. Nonetheless. the bulk of the delta- $V$ imparted to the vehicle would be expected from on-board pulse units. Thus, the increase in chemical explosive performance appears paramount to future EPPP research efforts.

\section{The ETO Booster Derivative}

Inexpensive access to space is the primary goal for NASA and the rocket community. Optimistically, EPPP might provide a cost effective, heavy lift vehicle alternative or its technologies parlayed into a more conventional internal. pulsed rocket engine. As alluded to earlier, the primary driver for this is the possibility of explosives with energy densities near or exceeding LOX LH2. But in addition. the EPPP vehicle would be radically different from the interplanetan fission pulsed system.

The obvious change is simply to use this new propellant in a conventional rocket chamber and nozzle. Depending on the characteristics of the propellant. that may not be effective or practical. Also. there is little chance that such a propellant would be significantly more powerful and be as cheap as LOX/LH2. This is a completely different application than discussed thus far. In this new function, the bottom line economic figures determine what is viable. Besides propellant costs, conventional liquid rockets enjoy a huge technology base and infrastructure. An EPPP booster would have to have some other special advantage over them.

The coupling of a high performance, beamed propellant mechanism and a modified EPPP vehicle has the potential to be a low cost booster. It would probably be expecting far to much for the entire ETO trip be accomplished by the beamed propellant EPPP system as explained earlier (i.e., exit velocities would be extremely high, atmospheric heating and drag are significant and overall efficiencies realistically could not beat a liquid upper stage). However, a substantial part of the flight might be economical with a reusable, carbon fiber, pusher plate/MTM booster. Instead of an inefficient flat plate (massive enough to withstand nuclear blasts), a half-sphere or parabolic bell shape, similar to a conventional rocket nozzle, would be used to obtain near ideal propellant coupling along with optimally shaped plasma pulses. The shock absorber, also massive on the interplanetary vehicle, would be almost eliminated or incorporated directly into the pusher cone structure. Only a small parachute recovery system need be added to the relatively lightweight, simplistic booster.

The pulse units would be mass-produced and use ultra small electronics for their detonation timing. All targeting would be done by the propellant supply mechanism, assuming dynamic control of each pulse unit during flight would be cost 
prohibitive. Dynamic control would also be slow compared to the intercept time for many of the pulses and add unnecessary complexity mass to each. The firing rate is a continual stream of pulses on the order of several a second to a known, nonevasive. large target area. Therefore, as the flight progresses. variations in atmospheric conditions. target position and the like. can be incorporated into the beamed propellant firing control to match required pulse velocity and direction. Variations outside this can be handled by active vehicle control over the detonation timing (i.e., earlier, later or aborted altogether). A large vehicle base (10 to 20 meters) offers an unusually large target that would significantly relax similar military aiming requirements. Even the pulse unit housing would be extremely minimal. such as a thin ablative protective layer. designed to survive the few seconds of flight time and easily break apart upon detonation, to avoid damaging the vehicle base with large fragments.

Keeping the vehicle and pulse units as simplistic as possible is keenly important to the economic competitiveness of the system. However. the gun launch system must be reliable and economical as well. Both the infrastructure and the power requirements to "beam" the propellant to the booster must not be excessive. Generally, the various beam propellant options appear to be in the same magnitude of complexity and expense as today's space launch systems. such as the Space Shuttle. The future. commercialized launch market will probably be more competitise. making the gun launch system costs even more pertinent.

\section{MARS MISSION ANALYSIS}

EPPP is well suited as a propulsion system for interplanetary missions. Manned interplanetary missions become increasingly feasible with propulsion systems that provide high lsp and thrust levels. EPPP is able to provide both these in abundance. The first manned interplanetary mission will likely be a Mars exploration mission. In this section, a Mars mission will be described at a cursory level, for a manned landing and return based upon an EPPP system employing today's technology.

\section{Key Mission Architecture Features}

This scenario is a first cut at a full Mars mission in less than I year. It is not optimized with respect to the capabilities of External Pulsed Plasma Propulsion (EPPP) or a GABRIEL series spacecraft. It merely uses the most realistic and reliable hardware that is available today to successfully conduct such a mission. Performance is expected to be enhanced if trades were done on departure dates. trajectories, payloads, mission timeline, crew size, alternate landers. The assumed EPPP performance values are considered practical using today's technology and some data suggests the actual values could be significantly better. Some ke! assumptions of the EPPP Mars mission Architecture are listed below:

\section{Crew size: 4}

2. Mission staging point: All staged elements are to be placed in LEO (standard Space Shuttle orbit. about $220 \mathrm{~nm}$ ) and autonomously dock together. Scenario based on June 3 . 2018 departure date.

3. Crew return transportation mode: The reentry/command pod is a multipurpose, aero-shield reentry module that serves as the Mars lander ascent decent crew compartment and GABRIEL's pulse operations command center. Note: A shuttle return from ISS maybe considered a mandatory requirement for a Mars mission as possible contamination, crew health and safety issues may preclude direct Earth reentry. However. on a strictly idealized propulsion scenario. a single capsule. ballistic reentr. is clearly the best option.

4. Insitu propulsion production option considered: None. Note: This is viewed as an other serious failure mode that can be mitigated with a craft that has "clean fuel", avoids autonomous machinery malfunctions, unsupervised. long-term storage of Mars propellants and possible added complexity for the crew once on the ground. It also allows emergency Mars surface evacuation at anytime from the moment they touch ground.

5. Auxiliary propulsion system type assumed: LOX CH + system on board GABRIEL. Note: More research needs to be given on the reliability of restarting LOX $\mathrm{CH} 4$ engines hundreds of times. Hydrazine and flywheels could be more attractive for this RCS application, but during pulsing. a strong, fast acting vector control mechanism is needed and a simple pressure feed $\mathrm{LOX} / \mathrm{CH} 4$ appears appropriate at this time.

6. Special crew or cargo in-space taxi requirements: None. The Earth reentry vehicle (reentry/command pod) is part of the Mars lander.

7. Special disposal requirements: None! - All nuclear pulse units are use in deep space and leave no elements that must be disposed (i.e. most particle remnants are going at solar system escape velocity). The vehicle itself should not be radioactive so no penalty is imposed for long-life. stable, disposal orbits after use (under the current scenario. GABRIEL is jettisoned before Earth reentry)

8. ETO transportation system for mission buildup: Unique ETO configuration for Momentum Transfer Mechanism (MTM) with Supporting Systems and Structures $\left(\mathrm{S}^{3}\right)$ attached to 4 Solid Rocket Boosters (SRB's). SRB's used for liftoff strapped to MTM/ $\mathrm{S}^{3}$. High Energy Chemical Pulse Units used for second phase of ascent and orbital insertion.

\section{Mars Mission Phase Event Timeline \& Metrics}

A typical mission timeline is presented in TABLE 3 . The sequence of events are adapted from typical Mars mission scenarios that minimizes the requirements of the main propulsive (i.e., single use vehicle and ballistic return capsule). Safety, reliability and cost were the key parameters that were considered in the selection and ordering of each event. as well as, what is presently technologically feasible. TABLE 4 includes some basic metrics on the EPPP system both for the chemical and fission operation modes. 
TABLE 3: MARS MISSION TIMELINE

\begin{tabular}{|c|c|c|}
\hline PHISE & ITEM \# & EVENT DESCRIPTION \\
\hline \multirow{11}{*}{ Deployment } & 1. & $\begin{array}{l}\text { Momentum Transfer Mechanism (.MTM) with Supporting Systems and Structures ( } S^{3} \text { ) launched into I.EO) } \\
\text { - + } \text { SRB }^{\circ} \text { s for initial lifioff for initial liftoff strapped to MTM } \mathrm{S}^{\prime} \\
\text { - High Energy Chemical Pulse Units (HECPU) used for second phase of ascent and orbital insertion. }\end{array}$ \\
\hline & 2. & $I^{51}$ Launch of HMM payload \\
\hline & 3. & AR\&D (Autonomous Rendezvous and Dock) of MTM $S^{3}$ and $1^{\text {st }} H M M$ payload and checkout activities. \\
\hline & 4. & $2^{\text {nd }}$ Launch of HMM payload \\
\hline & 5 & AR\&D (Autonomous Rendezvous and Dock) of MTM/ $S^{2}$ and $2^{\text {id }}$ HMM payload and checkout activities. \\
\hline & 6. & $3^{\text {cd }}$ Launch of HMM payload \\
\hline & 7 & AR\&D (Autonomous Rendezvous and Dock) of MTM $S$ and $3^{\text {rd }}$ HMM pavload and chechout activities. \\
\hline & 8. & 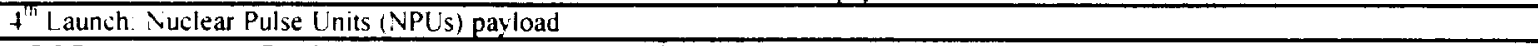 \\
\hline & 9. & AR\&D (Autonomous Rendezvous and Dock) of MTM/ $S^{\prime}$ and NPU payload and checkout activities. \\
\hline & 10. & Shuttle launch of 4-man crew. supplies and NPU arming parts \\
\hline & 11. & AR\&D (Autonomous Rendezvous and Dock) of MTM/S and Shuttle payload and checkout activities. \\
\hline \multirow{4}{*}{ Outbound } & 12. & Trans Mars Injection (TMI) pulse operation \\
\hline & 13. & Craft is rotated toward Mars (i.e. plate forward) \\
\hline & 14. & Crew inflates habitat and prepares for Mars landing during Earth to Mars transit. \\
\hline & 15. & $\begin{array}{l}\text { Mars Orhital Insertion (MOI) using NPUs. Mars insertion is achieved from NPU operation lafter habitat is refolded and left stored } \\
\text { until GABRIFL is traveling back to Earth) }\end{array}$ \\
\hline \multirow{4}{*}{ Vars } & 16. & $\begin{array}{l}\text { Single lander detaches and makes its descent with a drag chute then a nuclear thermal rocket engine soft landing All four } \\
\text { crewmembers are on the ground and GABRIEL. is left in low-energy idle mode (i.e. its main power supply being the bimodal nuclear } \\
\text { reactor on the lander). }\end{array}$ \\
\hline & 17. & $\begin{array}{l}\text { 31) das Mars itay is accomplished using lander as base station and small habitation module is unfolded for creat quarters stightly aw as } \\
\text { from the lander and its nuclear power supply. }\end{array}$ \\
\hline & 18 . & $\begin{array}{l}\text { Ascent stage leaves surface (landing leg structure. descent tankage. equipment. etc are left) and rendezvous with GABRIEL in lars } \\
\text { orbit. }\end{array}$ \\
\hline & 19. & Crew and Mars samples are in the reentry capsule when part of the ascent stage is jettisoned in orbit (i.e. the empty propellant tanks). \\
\hline \multirow{5}{*}{ Return } & 20. & $\begin{array}{l}\text { Since GABRIEL is ready to go (i.e. habitat is folded. crew in reentry/command pod. etc.) the NPUs are immediately used to leave } \\
\text { Mars orbit and insert onto an Earth trajectory. }\end{array}$ \\
\hline & 21. & Craft is rotated toward Earth (i.e. plate forward). \\
\hline & 22. & $\begin{array}{l}\text { Crew inflates habitat and exits reentry/command pod for } 7 \text {-month return flight during which they inspect. take samples and test the } \\
\text { EPPP system to gain further vehicle operation data before its release. }\end{array}$ \\
\hline & 23. & $\begin{array}{l}5 \text { days before Earth reentry and just beyond the minimum safe zone for fission pulse mode. all spare fission pulse units are expended. } \\
\text { The first one is ejected with the ascent stage bimodal nuclear power supply and both are completely vaporized (at a large distance } \\
\text { from the vehicle). The crew uses fuel cell battery backup for last power requirements. }\end{array}$ \\
\hline & 24. & $\begin{array}{l}\text { Crew enters the reentry/command pod. jettisons GABRIEL and reenters Earth from a bailistic trajectory. using a conventional heat } \\
\text { shield to slow down and parachute recovery into ocean. }\end{array}$ \\
\hline
\end{tabular}

\section{TECHNOLOGY SYNERGISM}

Many new technologies exist that can be easily modified for use in some aspect of EPPP. This is important to a new competitor in the rocket propulsion arena. Starting technology research programs in many different areas is time consuming and expensive. Resistance to another vehicle concept often comes from existing programs due to competitiveness and limited funding sources. However. EPPP technology needs should complement a wide variety of existing investigations from solid rocket research to solar sail studies. Figure 1 illustrates many of these synergistic technologies.

Benefits from material and structural development for existing rockets can be applied to the pusher plate and MTM system. Particularly important are carbon fiber composites. highly elastic structures and shock resistant materials.

A ternate EPPP approaches such as a canopy or umbrella design could become a reality using transmuted solar sail. tether. or inflatable optic technology know-how. Tethers themselves could be ideally employed in a transportation system where an EPPP booster's payload is inserted into orbit by a space-based tether. Or perhaps a large tether system would be used to transport a fission based interplanetary vehicle to beyond a safe operating zone.

All the fusion, antimatter and related ultra high-, power propulsion systems have direct or indirect research relationships. Any external driver system, be it lasers, plasma jets, electron / antimatter beams or other technology is a viable candidate for EPPP. Self-contained pulse units have some advantages, but an externally driven system usually allows smaller pulse yields with higher fuel burn up fractions and eliminates most political objections. Electromagnetic field interactions are extremely important research for plate protection. better plasma coupling and onboard power generation from each pulse. Fundamental physics 
TABLE 4: MARS EPPP METRICS

\begin{tabular}{|l|l|}
\hline \multicolumn{2}{|l|}{ Chemical Pulse Unit Performance } \\
\hline $\begin{array}{l}\text { Propulsion } \\
\text { system type }\end{array}$ & External Pulsed Plasma Propulsion (EPPP) \\
\hline Propellant used & $\begin{array}{l}\text { All-chemical. highly energetic explosives (i.e.. } \\
\text { HECPU) }\end{array}$ \\
\hline Thrust level & $\begin{array}{l}1.390 .000 \mathrm{Ibf} \text { average thrust level (assumes 500s } \\
\text { Isp. } 2 \text { minute SRB burn, 6 minute EPPP operation) }\end{array}$ \\
\hline Specific impulse & 400 s minimum \\
\hline $\begin{array}{l}\text { Engine system } \\
\text { thrust weight }\end{array}$ & $\begin{array}{l}1.13 \text { lbf/lbm (just after SRB separation. GABRIEL } \\
\text { and pulse units =1.230.000 lbs) }\end{array}$ \\
\hline Throttle range & $\begin{array}{l}\text { Variable (depends on the pulse unit frequency. } \\
\text { detonation distance from plate and vehicle } \\
\text { oscillatory modes) }\end{array}$ \\
\hline $\begin{array}{l}\text { Wultiple start } \\
\text { capability }\end{array}$ & Yes. (every pulse is a "restart"!) \\
\hline Reusable & Yes, for the rest of the Mars mission \\
\hline
\end{tabular}

\begin{tabular}{|c|c|}
\hline \multicolumn{2}{|r|}{ Fission Pulse Unit Performance } \\
\hline $\begin{array}{l}\text { Propulsion } \\
\text { System type }\end{array}$ & External Pulsed Plasma Propulsion (EPPP) in vacuum \\
\hline Propellant used & Fission based nuclear pulse units \\
\hline Thrust level & $100.000 \mathrm{lbf}$ (assumes 29 minute EPPP operation) \\
\hline Specitic impulse & $7500 \mathrm{~s}$ minimum \\
\hline $\begin{array}{l}\text { Engine sistem } \\
\text { thrust/ueight }\end{array}$ & $\begin{array}{l}0.18 \mathrm{lbf} / \mathrm{lbm} \text { ( for first pulsc. GABRIEL mass = } \\
542.652 \mathrm{lbm} \text { ) }\end{array}$ \\
\hline Throttle range & $\begin{array}{l}\text { Variable (depends on the pulse unit frequency. } \\
\text { detonation distance from plate and vehicle } \\
\text { oscillatory modes) }\end{array}$ \\
\hline $\begin{array}{l}\text { Multiple start } \\
\text { capability }\end{array}$ & Yes. (every pulse is a "restart"!) \\
\hline
\end{tabular}

investigations into plasma reactions in space and with materials are of interest as well.

Military research programs could supply the most numerous and important advantages to an EPPP program. Especially when considering that many of the military performance specifications are relaxed for EPPP utilization as discussed previously. Most notables are:

- Ultra high explosives without long term storage

- Blast resistant shielding with no fragmentation

- Accurate tracking/targeting of large, non-evasive vehicle

- Gun launch systems using large fixed installations

- High acceleration missiles with minimal electronic requirements

- Nuclear physics \& fission devices within existing database

\section{SUMMARY AND CONCLUSIONS}

A metamorphosis has begun for EPPP research. The original goals of a small, fast interplanetary vehicle $(5.000 \mathrm{sec}$. Isp and 5 meter diameter pusher plate) does not appear feasible using existing technologies. A minimum diameter of 15 meters is recommended for such a vehicle. Assembly or manufacture in orbit is expensive and risks quality, therefore. self-launching the wide plate system was considered the best option. Depending on future advances in high explosives. carrying ETO propellant might be possible. However. some form of beamed propellant technology was concluded as necessary during some portion of the ETO flight. Ground. aircraft and space-supplied propellant are possible. as well as. onboard chemical pulse units.

A mars scenario was presented for a 1 -year mission using existing technologies. No beamed propellant, tether or other advanced technology was used and the mission was not optimized for EPPP. Vehicle mass and ETO flight profile was similar to the Space Shuttle. After a 3 month outbound trip. the 4 man crew spends 30 days on the surface. Upon return the EPPP vehicle is jettisoned and a ballistic earth reentry is made in the command capsule. No other propulsion means is known which can perform the mission as fast and with as little mass in orbit.

A vast number of technologies can be applied to EPPP development. Many military and space propulsion systems complement EPPP, either in basic research or in system level component development. Advanced high explosive chemistry and beamed propellant are the two most significant technologies needed to be developed for a large EPPP vehicle launch.

\section{RECOMMENDATIONS AND FUTURE WORK}

The primary effort must be made to examine the potential for EPPP. The first is the communication to the many existing technology research entities the basic operational premises of external pulse operation and how their existing work can be of assistance to proving or disproving various EPPP approaches. Generation of published papers on specific EPPP aspects and in-depth trade studies of vehicle configurations are paramount. Unlike most space propulsion modes which have been studied extensively (i.e.. nuclear thermal, gas core, solar electric, advanced chemical to name a few), EPPP has had no direct research other than the 1958 ORION program and a dozen papers over the past 30 years. It should be a clear recommendation that the vast array of configurations EPPP can take, should be fully explored in future work by a variety of researchers, scientists and engineers.

Future work planned within the MSFC Propulsion Research Center is comprised of both system studies and experimental hardware testing. A more defined vehicle and mission analysis is planned to validate beamed propellant methodologies. The development of carbon fiber shock absorbers is underway and testing is expected in September 2000. A push for advanced chemical explosive research is expected to begin within the next year as well.

\section{ACKNOWLEDGMENTS}

Grateful acknowledgment is made to Peggy for proof reading under great time constraints. Support for the experimental hardware and the Mars mission scenario was obtained through NASA's Decadal Planning funding, as well as the MSFC Propulsion Research Center. 